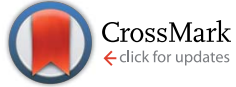

Cite this: RSC Adv., 2014, 4, 32438

Received 17th May 2014

Accepted 8th July 2014

DOI: $10.1039 / \mathrm{c} 4 \mathrm{ra0} 04651 \mathrm{j}$

www.rsc.org/advances

\section{Transition voltage spectroscopy of scanning tunneling microscopy vacuum junctions}

\author{
K. Sotthewes, $\uparrow^{\star}$ C. Hellenthal, $\uparrow^{\star}$ A. Kumar $\uparrow$ and H. J. W. Zandvliet \\ We have determined the dependence of the transition voltage (minimum in a $\ln \left(/ / V^{2}\right) v s . / / V$ plot) on the \\ vacuum gap width in ultra-high vacuum scanning tunneling microscopy junctions. We have performed \\ dual bias room temperature experiments with a $W$ tip and $\mathrm{Au}(111)$ as well as polycrystalline Pt surfaces. \\ For both type of surfaces the transition voltage decreases linearly with increasing inverse gap width. This \\ is in marked contrast with the standard models for quantum mechanical tunneling, which predict a linear \\ increase of the transition voltage with increasing inverse gap width. This remarkable discrepancy can \\ only be partly explained by the incorporation of an image charge effect and therefore there is a clear \\ need for a revision of the standard models for quantum mechanical tunneling in vacuum scanning \\ tunneling microscopy junctions.
}

\section{A Introduction}

The field of molecular electronics aims to investigate and realize elementary electronic devices relying on molecules for future elementary electronic devices. ${ }^{\mathbf{1}, \mathbf{2}}$ One of the essential parameters in charge transport through molecules is the location of the molecular energy levels with respect to the Fermi level., ${ }^{3,4}$ A wellestablished method to determine the electronic structure of an electrode-molecule-electrode junction is scanning tunneling spectroscopy, also referred to as current-voltage $I(V)$ spectroscopy. An increase in the current is observed when the Fermi level of one of the contacts lines up with a molecular energy level. The molecular levels that can be accessed by scanning tunneling spectroscopy typically lie in the range of several eVs around the Fermi level. Since the gap spacing in scanning tunneling microscopy is only $1 \mathrm{~nm}$ or less, the electric field strength can easily exceed $10^{9} \mathrm{~V} \mathrm{~m}^{-1}$.

Beebe et $a l .{ }^{5}$ introduced transition voltage spectroscopy (TVS) as another method to determine the tunneling barrier height $\phi$, which is the energy difference between the LUMO (lowest unoccupied molecular orbital) position and the Fermi level (for hole tunneling the barrier height is given by the energy difference between the HOMO (highest occupied molecular orbital) and the Fermi level). They proposed that the transition voltage $\left(V_{\mathrm{t}}\right)$, which is the minimum in a Fowler-Nordheim $(\mathrm{F}-\mathrm{N})$ plot, i.e. a plot of $\ln \left(I / V^{2}\right)$ versus $I / V$, provides direct information on the tunneling barrier height. Since the transition voltage is substantially smaller than the effective barrier height this

Physics of Interfaces and Nanomaterials, MESA ${ }^{+}$Institute for Nanotechnology, University of Twente, P.O. Box 217, 7500AE Enschede, The Netherlands. E-mail: k.sotthewes@utwente.nl; c.hellenthal@utwente.nl

$\dagger$ K. S., C. H and A. K. have contributed equally to this work. method allows one to study molecular levels at much smaller electric fields.

This interpretation of TVS was based on a picture of molecular junctions as tunnel barriers obeying the Simmons model for charge transport. ${ }^{6}$ They exemplify $V_{\mathrm{t}}$ to the point where the shape of the energy barrier, tilted by the applied bias voltage, changes from trapezoidal to triangular. The promise of determining the barrier height with such ease has led to a number of experimental ${ }^{4,7-12}$ and theoretical studies ${ }^{13-17}$ on TVS in molecular junctions. Later it was realized that, mathematically, the transition voltage is in fact a characteristic of pronounced nonlinear transport. Namely, it defines the point where the differential conductance is twice the pseudo-ohmic conductance $(\mathrm{d} I / \mathrm{d} V=2(I / V)) \cdot{ }^{18-22}$

Calculations of the tunneling current within the Simmons model challenged the validity of the barrier description for molecular junctions, ${ }^{19}$ leading to claims that $V_{\mathrm{t}}$ is not only related to the barrier height but it is also sensitive to other factors, such as the asymmetry of the junction and the molecular length (or tunneling distance). ${ }^{19,23}$ Based on these calculations it was suggested that TVS could be used as a tool to distinguish molecular junctions from vacuum junctions.

To check this assumption Trouwborst et al. ${ }^{24}$ investigated the distance dependence of the transition voltage of $\mathrm{Au}$-vacuum-Au mechanically controlled break junctions (MBJ). They observed that, contrary to the initial predictions, ${ }^{19}$ the experimental distance dependence on $V_{\mathrm{t}}$ in vacuum junctions is less pronounced than observed in molecular junctions. ${ }^{4}$ This weaker dependence was attributed to the image charge potential which lowers the barrier height for smaller vacuum gap widths. However, there is still a substantial difference between the experimental data and available theoretical predictions. For small gap widths, the effect of an image charge potential is 
smaller for a MBJ than for an STM junction because the MBJ vacuum junctions consists of two atomically sharp electrodes, ${ }^{25}$ whereas an STM junction is composed of one planar and one atomically sharp electrode. Given the fact that image potential effects are expected to be more pronounced in STM junctions it is worthwhile to perform TVS on an STM junction.

In this paper, we investigate the dependence of the transition voltage as a function of the gap width of a $\mathrm{W}$-vacuum-sample junction, using a tungsten (W) tip of an STM as one electrode and gold $(\mathrm{Au})$ or platinum $(\mathrm{Pt})$ substrate as the other electrode. In contrast to theoretical predictions, which predict a linear increase of the transition voltage with increasing inverse gap, we observed a linear decrease of the transition voltage. In addition, we found that the transition voltage, corresponding to the minima in the $\mathrm{F}-\mathrm{N}$-plot, changes from $1.8 \mathrm{~V}$ to $0.5 \mathrm{~V}$ as the vacuum gap width is reduced by $0.4 \mathrm{~nm}$. Such a dramatic variation of the transition voltage cannot be explained by the incorporation of an image charge potential in the popular and wellestablished Simmons model $^{6}$ as predicted by Huisman et al. ${ }^{19}$

\section{B Results and discussion}

Huisman et al. ${ }^{19}$ derived by reformulating the Stratton formula, ${ }^{26}$ a simple analytical expression for $V_{\mathrm{t}}$ given by,

$$
V_{\mathrm{t}}=\frac{2 \hbar}{e \sqrt{m}} \frac{\sqrt{2 \phi}}{d}
$$

where $e$ is the electronic charge, $m$ the electronic mass, $\phi$ is the tunneling barrier height and $d$ the tunnel barrier width in a simple square barrier model. They also showed that the difference between the Stratton and Simmons model is negligible, despite the fact that the Stratton approach is only an approximation. In the Simmons model the tunneling current is, for small voltages, given by,

$$
I \propto \frac{V}{d} \sqrt{\phi-\frac{e V}{2}} e^{-2 \frac{\sqrt{2 m}}{\hbar} d \sqrt{\phi-\frac{e V}{2}}} .
$$

The only 'free' variable parameter in this equation is the tunneling barrier height $\phi$.

Fig. 1 shows a number of $V_{\mathrm{t}}$ versus $1 / d$ plots as obtained from the Simmons model. As expected, a variation of $\phi$ does not have a large effect on the $V_{\mathrm{t}}$ versus $1 / d$ curves.

Fig. 2 shows an STM image recorded of the Pt sample as well as a set of $I-V$ measurements taken at a bias range from 2.5 to $-2.5 \mathrm{~V}$. The tip-substrate distance was varied by changing the set point current from $0.02 \mathrm{nA}$ to $40 \mathrm{nA}$ at a constant bias voltage of $2.5 \mathrm{~V}$. Increasing the current set point at a fixed bias voltage causes the tip-substrate distance to decrease. The variation of the tip-sample distance as a function of the current set point was determined through the use of $I-z$ measurements. These measurements indicate that changing the current set point from $0.02 \mathrm{nA}$ to $40 \mathrm{nA}$ results in a decrease in tip-substrate distance of $0.4 \mathrm{~nm}$. The $I-V$ measurements shown in Fig. 2B exhibit metal-vacuum-metal junction behavior, judging from the nonzero differential conductance values at zero bias. At

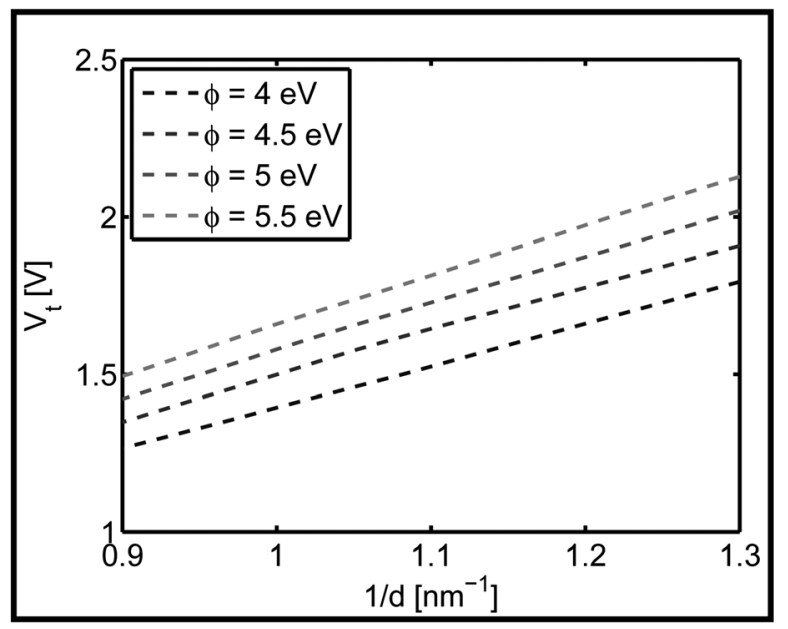

Fig. 1 Theoretically predicted $V_{\mathrm{t}}$ versus $1 / d$ graph for various barrier heights.

lower bias values, the slope of the $I-V$ curves behaves in a linear fashion, whereas for higher bias values the slope exhibits a rather steep increase. This trend can be further elucidated by plotting the $I-V$ curves in a Fowler-Nordheim representation, as depicted in Fig. 2C and D. The black circles refer to the minima of the $\mathrm{F}-\mathrm{N} I-V$ curves, which represents the transition voltage $V_{\mathrm{t}}$. From Fig. 2C it becomes immediately apparent that the transition voltage changes as a function of tip-sample separation. $V_{\mathrm{t}}$ increases with increasing gap width $d$. The value of $V_{\mathrm{t}}$ varies from $0.5 \mathrm{~V}$ at gap separations of approximately $0.8 \mathrm{~nm}$ to almost

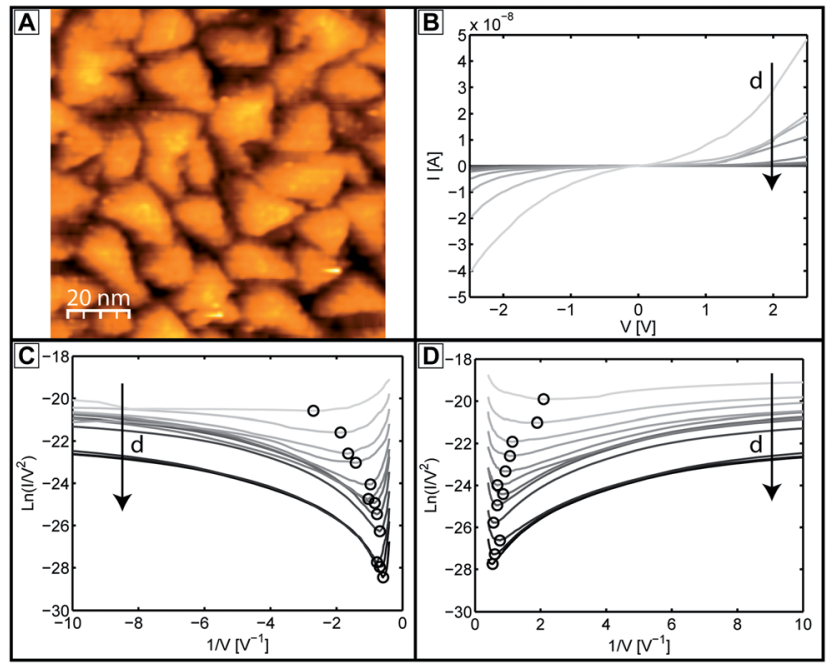

Fig. 2 (A) STM image of a polycrystalline Pt surface. (B) Currentvoltage $(I-V)$ measurements recorded on the Pt surface in the range from $+2.5 \mathrm{~V}$ to $-2.5 \mathrm{~V}$. The tip and the surface distance was varied by varying the set point current from $0.02 \mathrm{nA}$ to $40 \mathrm{nA}$ while keeping the sample bias constant at $+2.5 \mathrm{~V}$. The arrow points towards the direction of increasing vacuum gap width, $d$. ( $C$ and $D$ ) Fowler-Nordheim ( $F-N$ ) plot extracted from the current-voltage $(I-V)$ measurements shown in Fig. 2(B). For all vacuum gap widths a minimum can be observed in the $\mathrm{F}-\mathrm{N}$ plots, indicated by a circle. The arrow points toward the direction of increasing vacuum gap width, $d$. For larger set point currents (i.e. smaller $d$ ), the transition voltage is smaller and vice versa. 
$2.0 \mathrm{~V}$ at separations of $1.2 \mathrm{~nm}$. Furthermore, the transition appears to become sharper as the tip-sample separation increases, changing from a rather broad minimum at $0.8 \mathrm{~nm}$ to a sharp and well-defined minimum at separations of $1.2 \mathrm{~nm}$. No plateaus or double minima were observed in the $\mathrm{F}-\mathrm{N}$ plots.

Fig. 3 shows the same type of measurements for a $\mathrm{Au}(111)$ substrate. From the STM image, it is clear that the Au surface consists of large (111) oriented terraces separated by atomic steps. The $I-V$ curves (Fig. 3B) and $\mathrm{F}-\mathrm{N}$ curves (Fig. 3C and D) show the same general trend as those recorded on the $\mathrm{Pt}$ sample; $V_{\mathrm{t}}$ shifts to higher values and the transition becomes sharper as the tip-sample separation increases. The transitions, especially for small $d$, do appear to be a bit sharper than those observed for the Pt substrate.

In order to compare our results with the Stratton ${ }^{26}$ and Simmons models ${ }^{19}$ and previously conducted experiments on molecular break junctions, ${ }^{24}$ the minima of the $\mathrm{F}-\mathrm{N}$ plots (i.e. $V_{\mathrm{t}}$ ) were plotted versus $1 / \mathrm{d}$. Fig. 4 shows the results of the measurements performed on the Pt (Fig. 4A) and $\mathrm{Au}(111)$ (Fig. 4B) substrates. The absolute values of $V_{\mathrm{t}}$ for both the positive and negative bias ranges are plotted as a function of $1 / d$. This plot clearly shows that $V_{\mathrm{t}}$ varies between $1.8 \mathrm{~V}$ and $0.5 \mathrm{~V}$ in the range of $0.9 \mathrm{~nm}^{-1}$ to $1.3 \mathrm{~nm}^{-1}$. The most striking feature of Fig. 4 is the slope of the curves: the theory predicts a linear increase of $V_{\mathrm{t}}$ with increasing $1 / d$, whereas the experiments reveal a linear decrease of $V_{\mathrm{t}}$ with increasing $1 / d$. Additionally, the dependence of $V_{\mathrm{t}}$ on $1 / d$ is far stronger in the presented STS measurements $\left(1.5 \mathrm{~V}\right.$ in the range of $0.9 \mathrm{~nm}^{-1}$ to $\left.1.3 \mathrm{~nm}^{-1}\right)$ than found by Trouwborst et al. ${ }^{24}$ in their MBJ experiments ( $0.5 \mathrm{~V}$ over

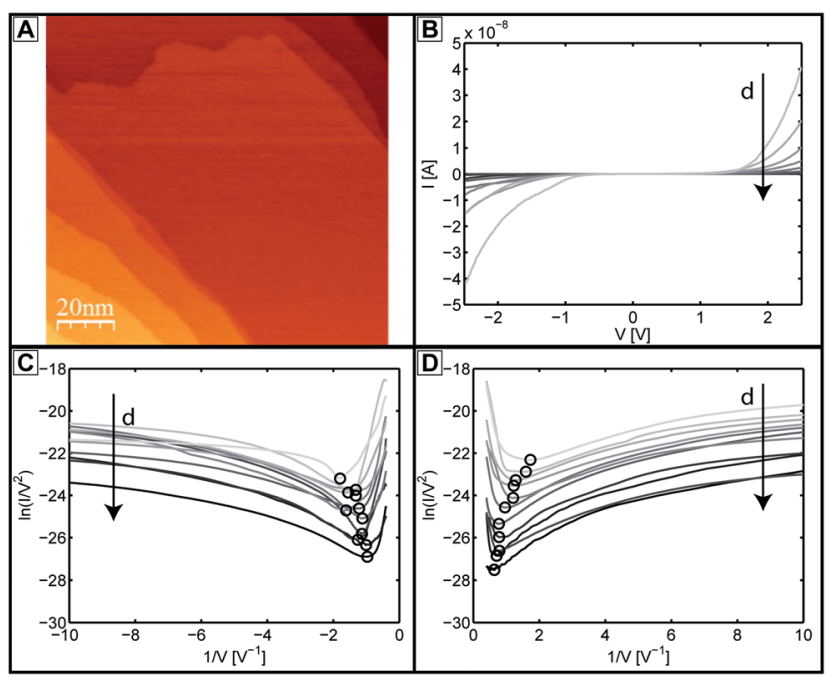

Fig. 3 (A) STM image of Au(111). (B) Current-voltage (I-V) measurements recorded on $\mathrm{Au}(111)$ in the range from $+2.5 \mathrm{~V}$ to $-2.5 \mathrm{~V}$. The tip and the surface distance was varied by varying the set point current from $0.02 \mathrm{nA}$ to $40 \mathrm{nA}$ while keeping the sample bias constant at +2.5 $\mathrm{V}$. The arrow points towards the direction of increasing vacuum gap width, $d$. ( $C$ and $D$ ) Fowler-Nordheim $(\mathrm{F}-\mathrm{N})$ plot extracted from the current-voltage $(I-V)$ measurements shown in Fig. 3(B). For all vacuum gap widths a minimum can be observed in the $\mathrm{F}-\mathrm{N}$ plots, indicated by a circle. The arrow points towards the direction of increasing vacuum gap width. For larger set point current (i.e. smaller d), the transition voltage is smaller and vice versa.

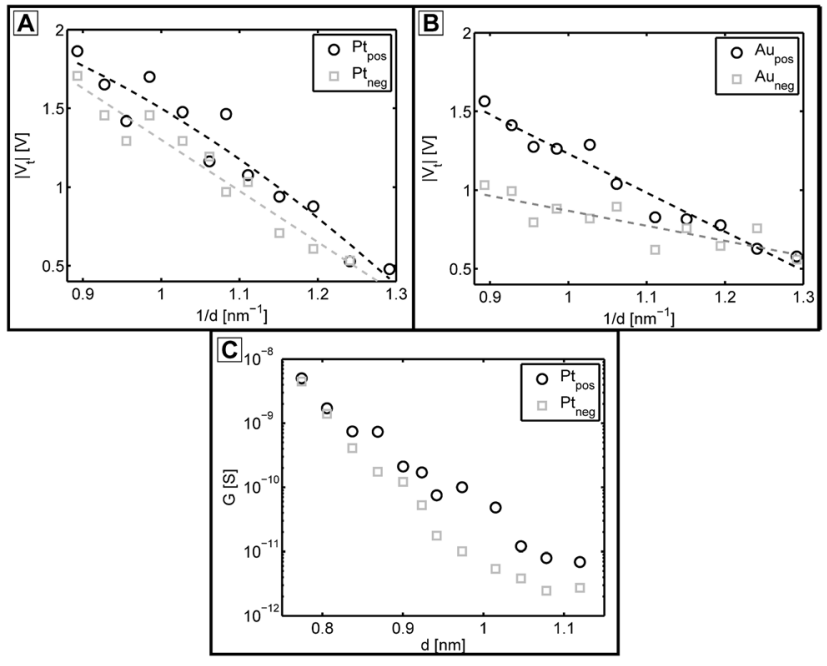

Fig. 4 (A) Absolute value of the transition voltage for Pt plotted versus $1 / d$. The transition voltage $V_{t}$ shows a linear $1 / d$ dependence. (B) Absolute value of the transition voltage for Au plotted versus 1/d. The transition voltage follows a linear trend for both positive and negative polarities, albeit with a significantly different slope. (C) Semilog plot of the conductance $G$ at the transition voltage $\left(G=I_{t} / V_{t}\right)$ versus $d$. At $d=1$ $\mathrm{nm}$ a transition from an exponential dependence to a non-exponential dependence is observed.

$0.6 \mathrm{~nm}^{-1}$ ). This observation calls into question previous claims that the magnitude of the absolute values of $V_{\mathrm{t}}$ can be used to distinguish vacuum junctions from molecular junctions.

The values of $V_{\mathrm{t}}$ for the Au sample at positive biases are slightly lower than those for Pt, which can be explained by the fact that Pt has a higher work function than Au. Interestingly, there is a substantial difference of the slope of the transition voltage of $\mathrm{Au}(111)$ versus $1 / d$ for positive and negative sample biases. We ascribe the decrease of the transition voltage of $\mathrm{Au}(111)$ at negative sample biases to the presence of a surface state located at $0.5 \mathrm{eV}$ below the Fermi level of $\mathrm{Au}(111) .{ }^{27}$

Another intriguing experimental observation is the distance dependence of the conductance at the transition point, i.e. $G_{\mathrm{t}}(=$ $I_{\mathrm{t}} / V_{\mathrm{t}}$ ), as a function of $d$ (see Fig. 4C). For small $d$ values an exponential decay is found, but at gap widths larger than $1 \mathrm{~nm}$ a crossover is found to a much weaker distance dependence. For the $\mathrm{Au}(111)$ samples only an exponential decay of the conductance is observed without any indication for the presence of a crossover.

In an attempt to explain the large discrepancy between the predicted and measured behavior of $V_{\mathrm{t}}$ as a function of $1 / d$, we will consider the effect of an image charge potential as has been suggested by Huisman et al. ${ }^{17}$ and Trouwborst et al. ${ }^{22}$ The existence of image charges ${ }^{6}$ can have an effect on the tunneling barrier height and width, as has been pointed out in previous studies. ${ }^{\mathbf{1 9 2 4}}$ To incorporate the effect of an image charge in the Simmons model, an extra term has to be added to the effective barrier height. The mean value of the potential barrier height is then given by:

$$
\bar{\phi}=\phi_{0}-\frac{e V\left(d_{2}-d_{1}\right)}{2 d}-1.15 \zeta \frac{e^{2} \ln (2) d}{16 \pi \varepsilon_{0}\left(d_{2}-d_{1}\right)} \ln \left(\frac{d_{2}(d-d)_{1}}{d_{1}\left(d-d_{2}\right)}\right) .
$$




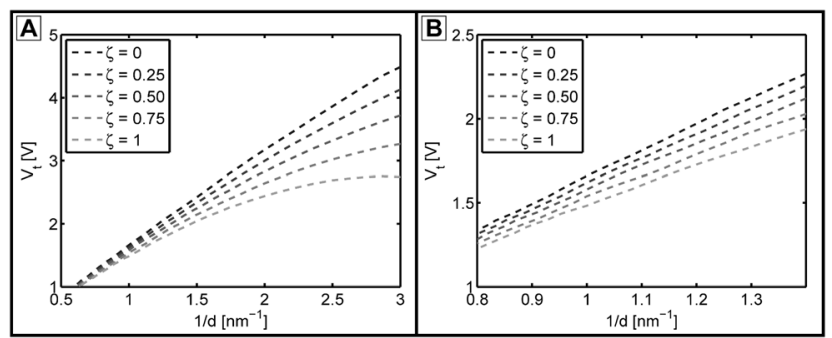

Fig. 5 The effect of an image charge potential $\zeta=0.25,0.50,0.75$ and 1) (A) for an extended $1 / d$ range and (B)for an $1 / d$ range typical to our STS experiments.

When eqn (3) is substituted into eqn (2), we find the following expression for the current

$$
I \propto \frac{V}{d} \sqrt{\bar{\phi}} e^{-2 \frac{\sqrt{2 m}}{\hbar} d \sqrt{\bar{\phi}}} .
$$

Here, $d_{1}$ is the distance between the potential barrier at the Fermi level of the tip and the vacuum, and $d_{2}$ is the distance from the vacuum to the potential barrier of the sample. Thus the barrier width at the Fermi level decreases from $d$ to $d_{2}-d_{1} . \zeta$ varies between 0 for a system consisting of two atomically sharp tips (i.e. a break junction) and 1 for a system consisting of two parallel plates. For all non-zero values of $\zeta$, the image charges will lower the effective barrier, and thus cause the value of $V_{\mathrm{t}}$ to drop as well. Because the impact of the image charges is also dependent on the tip-substrate separation, a non-zero value for $\zeta$ will also introduce a curvature in the $V_{\mathrm{t}}$ versus $1 / d$ plot for sufficiently small values of $d$. Fig. $5 \mathrm{~A}$ shows that for high values of $\zeta$, the slope of the $V_{\mathrm{t}}$ versus $1 / d$ plot will eventually change sign. However, this only happens for values of $1 / d$ that are substantially larger than the typical STS gap widths. Fig. 5B shows the effect of different $\zeta$ values for the $1 / d$ ranges that were typically encountered in our experimental STS study. At these gap widths, $\zeta$ has only very little effect on the transition voltage aside from a small offset. Therefore we have to conclude that image charges cannot explain the negative slopes found in our experimental $V_{\mathrm{t}}$ versus $1 / d$ plots. As such, we are forced to conclude that the current Simmons model does not capture the quantum mechanical tunneling process of a STM junction perfectly. At this stage we have no clue how to modify or extend the Simmons model to improve the agreement between experiment and theory. The interested reader is referred to a companion paper in the same issue by I. Baldea..$^{28}$

\section{Experimental}

The experiments were carried out in an ultra-high vacuum (UHV) Omicron room-temperature scanning tunneling microscope (STM 1). The Pt substrates were prepared by physical vapor deposition of $200 \mathrm{~nm}$ Pt on a Si substrate resulting into a

\$ This manuscript is a companion paper and is intended to be read in conjunction with the manuscript DOI: 10.1039/c4ra04648j by I. Baldea. granular structure. Au substrates $\left(11 \times 11 \mathrm{~mm}^{2}, 250 \mathrm{~nm}\right.$ Au on 2 $\mathrm{nm} \mathrm{Cr}$ on borosilicate glass) for STM measurements were purchased from Arrandee (Werther, Germany). Au(111) samples were obtained by annealing the substrates in a high purity $\mathrm{H}_{2}$ flame for $5 \mathrm{~min}$. The measurements were performed using a $\mathrm{W}$ tip prepared by electrochemical etching. In total approximately $10000 I-V$ curves were recorded on each substrate. The $I-V$ measurements were recorded with the tip at a predefined gap width. The feedback loop was switched off and a voltage ramp $(+2.5$ to $-2.5 \mathrm{~V})$ was applied with a typical voltage step size of 15 $\mathrm{mV}$. In order to remove the offset of the $I V$ converter the current at zero bias was set to zero for each $I-V$ trace. Distance dependent $I-V$ measurements were realized by changing the set point current at a constant sample bias voltage. The sample bias voltage was kept at the same value as the start voltage of the $I-V$ measurement to avoid any capacitive induced artifacts. We performed current-distance $(I-z)$ spectroscopy to determine the relative separation between the tip and substrate. A fraction $(5 \%)$ of the $I-V$ curves were excluded from the analysis because of the presence of current peaks induced by instabilities at larger tunneling currents, i.e. smaller substrate-tip separations.

\section{Conclusions}

We have determined the dependence of the transition voltage (minimum in a Fowler-Nordheim plot) on the vacuum gap width in ultra-high vacuum scanning tunneling microscopy junctions. In contrast to theoretical predictions the transition voltage does not increase, but decrease, with increasing inverse gap width. Including the effects of image charges in the standard Simmons model is insufficient to account for this discrepancy, indicating the need for further experimental and theoretical study to determine the exact cause of this behavior.

\section{Acknowledgements}

We would like to thank Dr Baldea for many valuable comments and suggestions. Financial support for this work provided by the Dutch Organization for Fundamental Research (FOM, 11PR2900) and the Dutch Organization for Scientific Research (NWO/CW ECHO.08.F2.008) is gratefully acknowledged.

\section{References}

1 S. J. van der Molen and P. Liljeroth, J. Phys.: Condens. Matter, 2010, 22, 133001.

2 K. Sotthewes, V. Geskin, R. Heimbuch, A. Kumar and H. J. W. Zandvliet, APL Mater., 2014, 2, 010701.

3 S. H. Choi, B. Kim and C. D. Frisbie, Science, 2008, 320, 1482.

4 J. M. Beebe, B. Kim, C. D. Frisbie and J. G. Kushmerick, ACS Nano, 2008, 2, 827.

5 J. M. Beebe, B. Kim, J. W. Gadzuk, C. D. Frisbie and J. G. Kushmerick, Phys. Rev. Lett., 2006, 97, 026801.

6 J. G. Simmons, J. Appl. Phys., 1963, 34, 1793.

7 L. H. Yu, N. Gergel-Hackett, C. D. Zangmeister, C. A. Hacker, C. A. Richter and J. G. Kushmerick, J. Phys.: Condens. Matter, 2008, 20, 374114. 
8 N. Bennett, G. Z. Xu, L. J. Esdaile, H. L. Anderson, J. E. Macdonald and M. Elliott, Small, 2010, 6, 2604.

9 M. C. Lennartz, N. Atodiresei, V. Caciuc and S. Karthauser, J. Phys. Chem. C, 2011, 115, 15025.

10 G. Wang, Y. Kim, S. I. Na, Y. H. Kahng, J. Ku, S. Park, Y. H. Jang, D. Y. Kim and T. Lee, J. Phys. Chem. C, 2011, 115, 17979.

11 S. Y. Guo, J. Hihath, I. Diez-Perez and N. J. Tao, J. Am. Chem. Soc., 2011, 133, 19189.

12 G. Ricoeur, S. Lenfant, D. Guerin and D. Vuillaume, J. Phys. Chem. C, 2012, 116, 20722.

13 T. Markussen, J. Z. Chen and K. S. Thygesen, Phys. Rev. B: Condens. Matter Mater. Phys., 2011, 83, 155407.

$14 \mathrm{~K}$. L. Wu, M. L. Bai, S. Sanvito and S. M. Hou, Nanotechnology, 2013, 24, 025203.

15 J. Z. Chen, T. Markussen and K. S. Thygesen, Phys. Rev. B: Condens. Matter Mater. Phys., 2010, 82, 121412(R).

16 I. Baldea, J. Phys. Chem. Solids, 2012, 73, 1151.

17 K. L. Wu, M. L. Bai, S. Sanvito and S. M. Hou, J. Chem. Phys., 2013, 139, 194703.
18 I. Baldea, J. Am. Chem. Soc., 2012, 134, 7958.

19 E. H. Huisman, C. M. Guedon, B. J. van Wees and S. J. van der Molen, Nano Lett., 2009, 9, 3909.

20 A. Vilan, D. Cahen and E. Kraisler, ACS Nano, 2013, 7, 695.

21 M. Araidai and M. Tsukada, Phys. Rev. B: Condens. Matter Mater. Phys., 2010, 81.

22 I. Baldea, Chem. Phys., 2012, 400, 65-71.

23 F. Mirjani, J. M. Thijssen and S. J. van der Molen, Phys. Rev. B: Condens. Matter Mater. Phys., 2011, 84, 115402.

24 M. L. Trouwborst, C. A. Martin, R. H. M. Smit, C. M. Guedon, T. A. Baart, S. J. van der Molen and J. M. van Ruitenbeek, Nano Lett., 2011, 11, 614.

25 I. Baldea and H. Koppel, Phys. Status Solidi B, 2012, 249, 1791.

26 R. Stratton, J. Phys. Chem. Solids, 1962, 23, 1177.

27 W. Chen, V. Madhavan, T. Jamneala and M. F. Crommie, Phys. Rev. Lett., 1998, 80, 1469.

28 I. Baldea, DOI: 10.1039/c4ra04648j. 